\title{
CHARACTERIZATION OF THE SCATTERED FIELD BY AN URBAN AREA IN THE X-FREQUENCY BAND FOR BISTATIC AND MONOSTATIC RADAR CONFIGURATIONS
}

\author{
Nguyen Ngoc Truong Minh, David Lautru, Hélène Roussel \\ UPMC Univ Paris 6, UR2, L2E, BC 252 \\ 4 place Jussieu, F-75005, Paris, France \\ Tel: +33 (0)144-274-386 ; Email: ngoc.nguyen14@etu.upmc.fr
}

\section{INTRODUCTION}

SAR (Synthetic Aperture Radar) imaging of urban areas is an interesting topic which has been developped for many recent years with some promising studies [1][2]. This paper proposes a model to characterize the scattered field by an urban area for bistatic and monostatic radar configurations. In mode bistatic, the radars are designed to operate with two separate antennas to perform the transmit and the receive functions (see Fig. 1). This model is validated in the frequency range 8-12 GHz (X-band), but can be applied in Ku-frequency band. The calculation of the scattered field is based on the Ray tracing combined with the Uniform Theory of Diffraction (UTD). Our model allows us to evaluate at any point the amplitude and phase of the diffracted field by a group of buildings. The applications of this model are not only the detection of moving targets in urban areas but also the analysis of electromagnetic wave propagation in this environment.

We chose this general configuration in a way to improve the radar capacity of detection and identification, and thus to increase the detection capacity of a target [3]. To evaluate the best bistatic configuration, it is necessary, in the first step, to develop a model to predict the diffracted field by an urban area. Many models have been already proposed for the propagation prediction in urban medium [4] [5]. The aim of this present work is to assess the quality of a relation (i.e between the transmitter and the receiver) according to the main parameters of urban environments (density of buildings, height of buildings ... ).

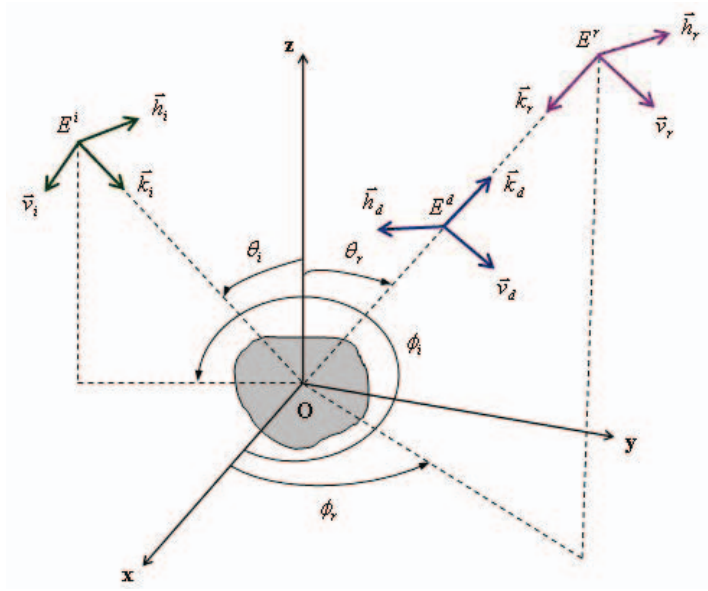

Fig. 1. Configuration of the mode BiSAR

\section{THE MODEL}

To develop a realistic model, we must on one hand define a canonical representation of an urban area similar to the reality and on the other hand an accurate electromagnetic model inserting the main interactions involved in urban areas. Due to these two criteria, a model based on the Ray tracing combined with the Uniform Theory of Diffraction (UTD) is proposed to determine the diffracted field by an urban area illuminated by a plane wave in the high frequency band $(8-12 \mathrm{GHz})$. 


\subsection{Representation of an urban area}

To build up an electromagnetic model inserting all the possible interactions between the incident wave and the elements in the observed zone, we chose a general representation of urban areas. Indeed, this representation is justified in considering that urban objects (buildings, cars ...) are always much more larger than the incident wavelengths (2.4-3.6 cm for X-band). Furthermore, the roughness of the ground is not taken into account because it is small compared to the incident wavelength. The buildings and the streets are represented by parallelepipeds placed on a dielectric plane interface representing the interface air/ground (see Fig. 2). The permittivities of the buildings and the ground depend on the moisture and defined by the real and imaginary parts of its complex relative permittivities $\epsilon_{b}$ and $\epsilon_{r}$. In this study, the relative permittivities of buildings and ground are equal to those of concrete $\left(\epsilon_{b}=7.3-j * 0.36\right)$ and soil $\left(\epsilon_{r}=28.5-j * 12.8\right)$ and their relative permeabilities are equal to one. The buildings are placed at deterministic positions on a $L_{x} \times L_{y}$-side parcel.

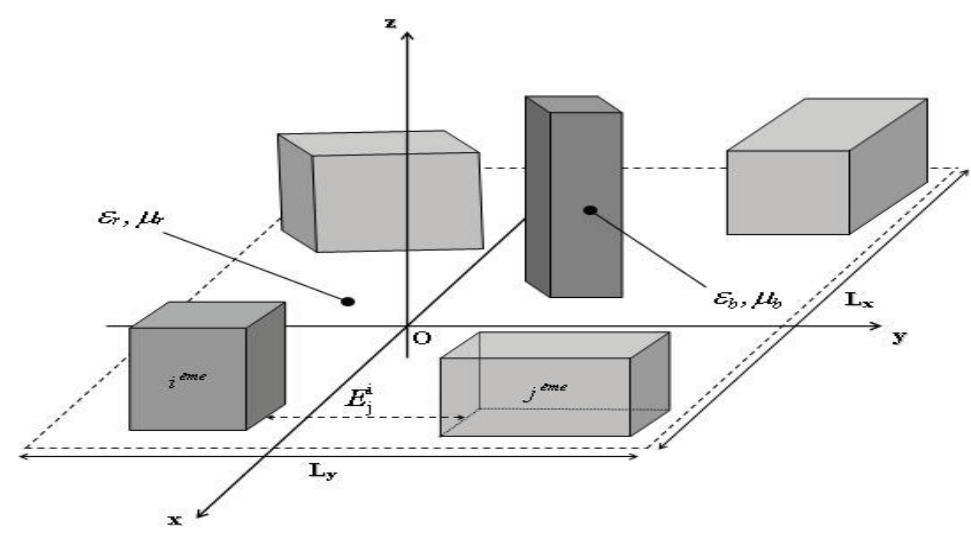

Fig. 2. Representation of a $L_{x} \times \mathrm{L}_{y}$ urban area

\subsection{Ray tracing}

A ray-tracing method is used to establish the geometrical path of the rays inside the illuminated scenarios (urban areas). However, in order to integrate all the diffractions on the edges of the buildings, we should reduce the intervals between the rays and as a consequence increase the time of calculation. To avoid it, we have developped a hybrid ray-tracing method which can refine the mesh in the inhomogeneous areas (object edges, edge diffraction ....). This adaptive subdivision adjusts the distance between two adjacents rays to find the intersection with the edges. A pre-define criteria of diffraction is needed when we use this method [6].

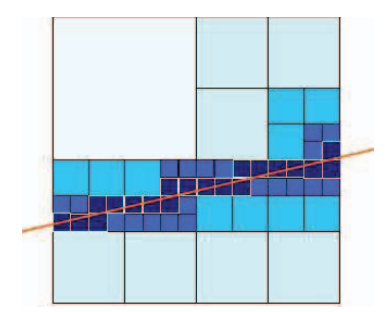

Fig. 3. For exemple, the hybrid ray-tracing method is used to find the intersections between a four-ray rectangular and an edge of a building after four times subdivions

\subsection{Calculation of the diffracted field}

When we consider a reflection at a point $O$, the reflected field at a point $M$ (after reflection) is obtained as a function of the transmitted field, reflection coefficients and propagation delay :

$$
\vec{E}^{r}(M)=\vec{E}^{r}(O) e^{-j \overrightarrow{k_{r}} \overrightarrow{O M}}=\left\{E_{v}^{r}(O) \overrightarrow{v_{r}}+E_{h}^{r}(O) \overrightarrow{h_{r}}\right\} e^{-j \overrightarrow{k_{r}} \overrightarrow{O M}}=\left\{R_{v} E_{v}^{i}(O) \overrightarrow{v_{r}}+R_{h} E_{h}^{i}(O) \overrightarrow{h_{r}}\right\} e^{-j \overrightarrow{k_{r}} O \vec{M}}
$$


where $R_{v}, R_{h}$ are the Fresnel vertical and horizontal reflection coefficients. In the case of diffraction at a point $O$ (intersection of a ray with an edge of a building), the formulation of the diffracted field at a point $M$ (after diffraction) is expressed by using the Uniform Theory of Diffraction [7] :

$$
\vec{E}^{d}(M)=E_{v}^{d}(M) \overrightarrow{v_{d}}+E_{h}^{d}(M) \overrightarrow{h_{d}}=A\left\{D_{v} E_{v}^{i}(O) \overrightarrow{v_{d}}+D_{h} E_{h}^{i}(O) \overrightarrow{h_{d}}\right\} e^{-j \overrightarrow{k_{d} O M}}
$$

where $D_{v}, D_{h}$ are the vertical and horizontal diffraction coefficients and A is the geometric divergence factor. Finally, the total field at a given point $M$ (the receiver) will be obtained after multiple reflections and/or one diffraction. For higher orders of diffraction, we consider that the signal is weak enough to be neglected.

\section{RESULTS}

We will now consider a configuration of four buildings. The heights of the buildings are respectively $\mathrm{H}=\left[\begin{array}{llll}8 & 14 & 10 & 12\end{array}\right]$ (m), the

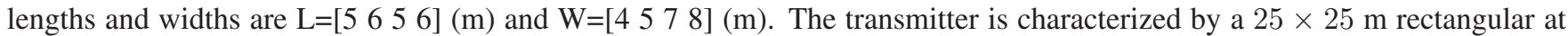
$\theta_{i}=28^{\circ}$ and $\phi_{i}=70^{\circ}$ and is at a height of 500m, the receiver is a $1 \times 1 \mathrm{~m}$ rectangular aperture moving on a half-sphere of radius $700 \mathrm{~m}$. The amplitude of the transmitted electric field is $1 \mathrm{~V} / \mathrm{m}$. Fig. 4 gives us (a) the configuration of the simulation and (b) the ray diffusion when the incident wave illuminates the considered group of buildings. The rays in blue are the direct diffracted rays while the green ones are the pure multiples reflections or a diffraction followed by multiples reflections.

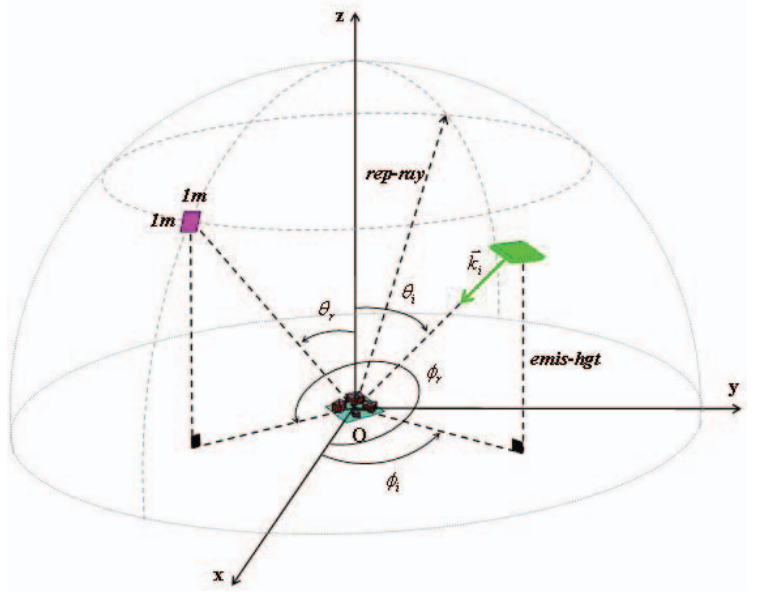

(a) Configuration of buildings and radars

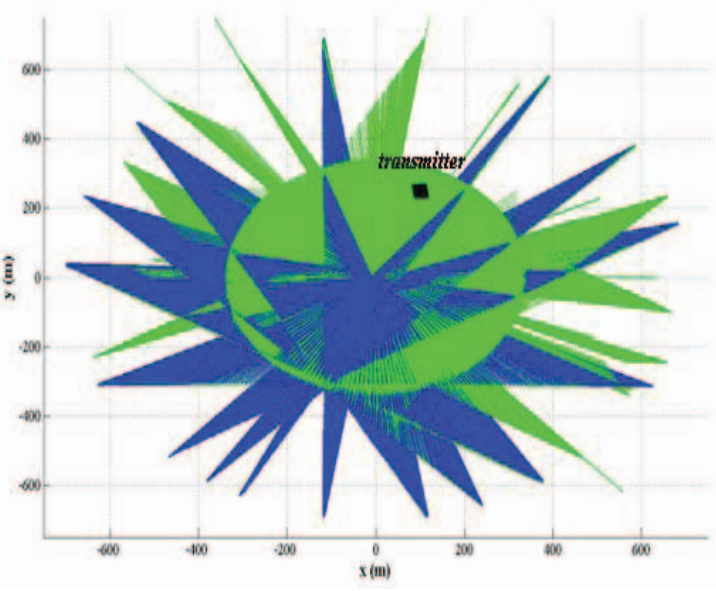

(b) Visualisation of ray diffusion

Fig. 4. Simulation for a composition of four buildings

In Fig. 5, we represent the received power of the diffracted field for both co-polar (V-V, H-H) and cross-polar (H-V, V-H) in the same range from $-250 \mathrm{~dB}$ to $0 \mathrm{~dB}$. We note that the main contributions come from the field due to multiple reflections and the level of the diffracted rays is much more smaller. Furthermore, there are some areas where the field is stronger than others because they receive the reflections on the roofs, ground and wall surfaces. The maximum is found in the specular direction $\left(\theta_{r}=28^{\circ}\right.$ and $\left.\phi_{r}=250^{\circ}\right)$. Beside that, the continuous parabolic curves are due to the direct diffractions by the roofs and wall surfaces while the discontinuous curves are due to the diffractions that are followed by multiple reflections. According to Fig. 5, we can see the level for $\mathrm{H}-\mathrm{H}$ polarization is lower than for $\mathrm{V}-\mathrm{V}$. Moreover, cross-polar $(\mathrm{H}-\mathrm{V}$ and $\mathrm{V}-\mathrm{H})$ are lower than co-polar (V-V and H-H).

\section{CONCLUSION}

We have defined a model to characterize the scattered field by an urban area in the X-band. This field depends on the polarization of the incident wave, the geometry and the materials composing of the buildings and the ground. With our model, the diffracted field by an urban area is obtained for a deterministic distribution of buildings. To obtain an average value of the diffracted field in a given direction it should be calculated for several possible configurations and take the mean value. The applications are to evaluate the quality of the diffracted field according to the parameters of the urban environment and to study of the attenuation of a wave propagation in this medium. 


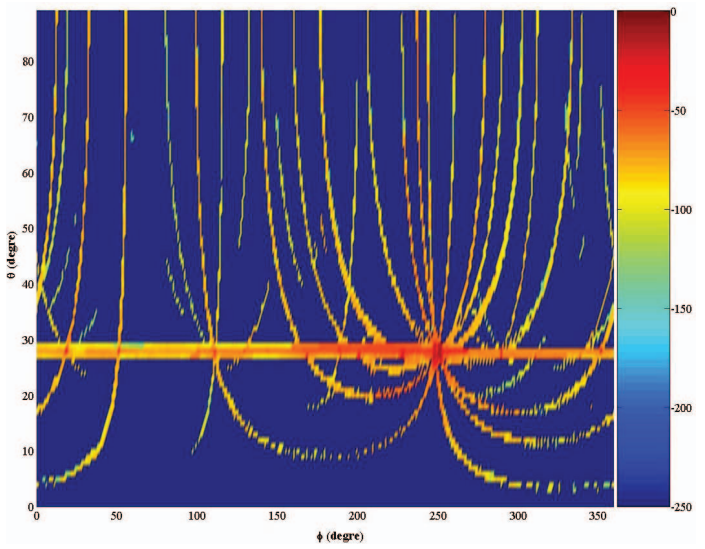

(a) Co-polar V-V

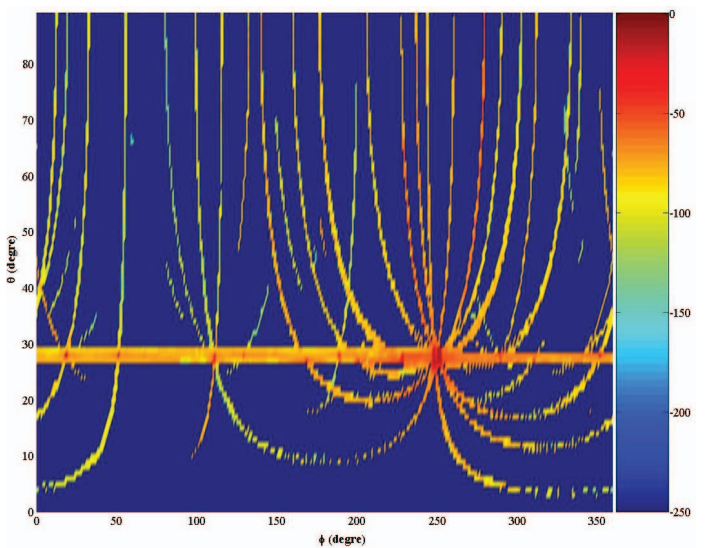

(c) Co-polar $\mathrm{H}-\mathrm{H}$

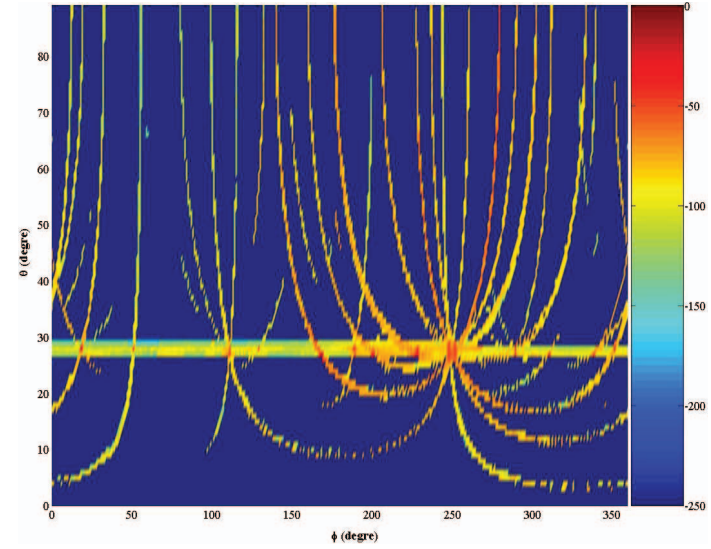

(b) Cross-polar H-V

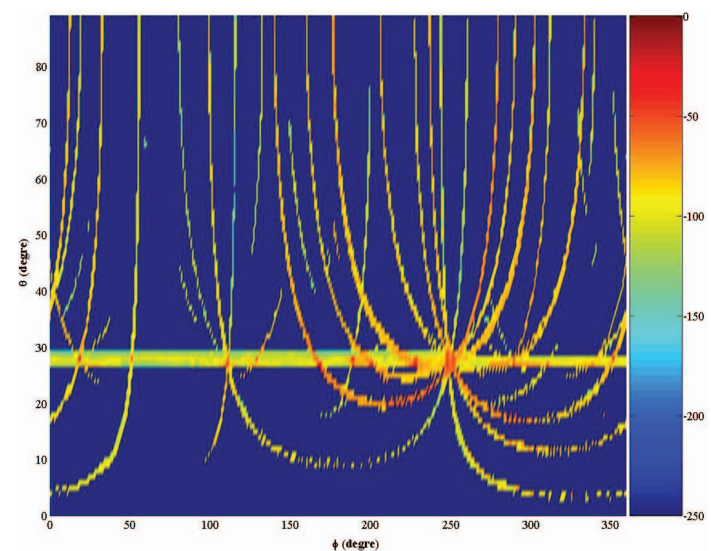

(d) Cross-polar V-H

Fig. 5. Received power for two polarizations in $\mathrm{dB}$

\section{REFERENCES}

[1] Christoph H. Gierull, "Statistical analysis of multilook sar interferograms for cfar detection of ground moving targets," IEEE TRANSACTIONS ON GEOSCIENCE AND REMOTE SENSING, vol. 42, No. 4, pp. 691-701, April 2004.

[2] Xavier Dupuis Philippe Paillou Franck Garestier, Pascale Dubois-Fernandez and Irena Hajnsek, "Polinsar analysis of xband data over vegetated and urban areas," IEEE TRANSACTIONS ON GEOSCIENCE AND REMOTE SENSING, vol. 44, No. 2, pp. 356-364, February 2006.

[3] M. J. Ben Kassem and A. Khenchaf, "Bistatic mapping radar bisar," OCEANS 2003. Proceedings, vol. 5, pp. 2754-2760, September 2003.

[4] Vittorio Degli-Esposti, "A diffuse scattering model for urban propagation prediction," IEEE TRANSACTIONS ON ANTENNAS AND PROPAGATION, vol. 49, No. 7, pp. 1111-1113, July 2001.

[5] Florence Tupin Céline Tison, Jean-Marie Nicolas and Henri Maître, "A new statistical model for markovian classification of urban areas in high-resolution sar images," IEEE TRANSACTIONS ON GEOSCIENCE AND REMOTE SENSING, vol. 42, No. 10, pp. 2046-2056, October 2004.

[6] Emmanuelle CONIL, Propagation électromagnétique en milieu complexe du champ proche au champ lointain, Ph.D. thesis, INSTITUT NATIONAL POLYTECHNIQUE DE GRENOBLE, Novembre 2005.

[7] Robert G. Kouyoumjian and Prabhakar H. Pathak, "A uniform geometrical theory of diffraction for an edge in a perfectly conducting surface,” PROCEEDINGS OF THE IEEE, vol. 62, No. 11, pp. 1448-1461, November 1974. 